\title{
Lusioersily
}

\section{Investigation of the relationship between macular pigment levels and rod-mediated dark adaptation in intermediate AMD.}

Beirne, R., \& McConnell, E. (2019). Investigation of the relationship between macular pigment levels and rodmediated dark adaptation in intermediate AMD. Dark adaptation and macular pigment in AMD. Clinical and Experimental Optometry, 102(6), 611-616. https://doi.org/doi: 10.1111/cxo.12882

Link to publication record in Ulster University Research Portal

\section{Published in:}

Clinical and Experimental Optometry

Publication Status:

Published (in print/issue): 30/11/2019

DOI:

doi: $10.1111 /$ cxo. 12882

\section{Document Version}

Author Accepted version

\section{General rights}

Copyright for the publications made accessible via Ulster University's Research Portal is retained by the author(s) and / or other copyright owners and it is a condition of accessing these publications that users recognise and abide by the legal requirements associated with these rights.

\section{Take down policy}

The Research Portal is Ulster University's institutional repository that provides access to Ulster's research outputs. Every effort has been made to ensure that content in the Research Portal does not infringe any person's rights, or applicable UK laws. If you discover content in the Research Portal that you believe breaches copyright or violates any law, please contact pure-support@ulster.ac.uk. 


\section{RESEARCH}

An investigation of the relationship between macular pigment levels and rod-mediated dark adaptation in intermediate AMD.

Raymond O. Beirne PhD BSc (Hons) MCOptom PG Cert Med Ret

Emma McConnell BSc (Hons) MCOptom PG Cert Low Vision

Vision Science Research Group, School of Biomedical Sciences, University of Ulster, Coleraine, BT52 1SA, Northern Ireland, United Kingdom.

Corresponding Author: Dr Raymond Beirne

E-mail: r.beirne@ulster.ac.uk

Tel: +44(0) 2870124477

Running title: Dark adaptation and macular pigment in AMD.

Keywords: age-related macular degeneration, macular pigment, rods

Word count: 3596 
Background: It has been shown that rod-mediated dark adaptation is significantly delayed in ageing, a change which is exacerbated in age-related macular degeneration. Levels of lutein and zeaxanthin, the two main constituents of macular pigment have been found in rod outer segments, indicating that the macular pigment may have an influence on rod-mediated dark adaptation. The aim of this study was to determine if rod-mediated dark adaptation is associated with central macular pigment levels in individuals with intermediate stage age-related macular degeneration.

Methods: A cross sectional observational study included individuals with acuity better than 6/15 Snellen and intermediate stage age-related macular degeneration based on graded fundus photographs using an internationally accepted grading scale. Rod-mediated dark adaptation was assessed at five degrees eccentricity in the superior retina (inferior visual field) using the rod intercept time measure from the MacuLogix AdaptDx. Macular pigment optical density was measured at 0.5 degrees eccentricity using a heterochromatic flicker photometry-based method.

Results: Twenty-seven individuals (mean age 76.7 years) with intermediate stage age-related macular degeneration and twenty-three age-matched normal controls (mean age 74.0 years) were recruited. Rod-mediated dark adaptation was significantly delayed in intermediate stage age-related macular degeneration compared with healthy controls (32.9 mins versus 10.7 mins, $\mathrm{p}<0.01)$. There was no statistically significant correlation between the rod intercept time and the level of macular pigment in those with intermediate age-related macular degeneration $(r=-.04$, $\mathrm{p}=0.85$ ).

Conclusion: The results did not support the hypothesis that higher macular pigment is associated with improved rod-mediated performance or that higher levels of macular pigment protect rod-mediated function in intermediate age-related macular degeneration. 
One extremely informative measure of visual function in the ageing eye is the dark adaptation curve, which is a measure of the ability of the eye to adapt to the dark after the retina has been bleached with a bright light. ${ }^{1}$ The dark adaptation curve has two basic components, the first is cone mediated and the second is rod mediated. The curve displays significant changes in the healthy ageing eye ${ }^{2}$ that are exacerbated in age-related macular degeneration (AMD). ${ }^{3-10}$ Almost all studies with AMD participants have shown that rod mediated dark adaptation (RMDA) is significantly increased in comparison to age-matched individuals free from disease. This is seen in a delayed rod-cone break time and a shallower slope of the rod-mediated portion of the curve. ${ }^{4,6,8}$ For examples of this change see Figure 1 in Owsley et al., 2007, ${ }^{4}$ and Figure 2 in Flamendorf et al., $2015 .^{8}$ It has been postulated that this deficit in RMDA with age and AMD is principally caused by impaired translocation of retinoids and other essentials from the choroid across the retinal pigment epithelium - Bruch's membrane sub-retinal space. ${ }^{11}$ This dysfunction is heightened in AMD through the accumulation of drusen and retinal pigment epithelium pigmentation changes. ${ }^{11}$ An age-related deterioration in the optical quality of the eye may also contribute to any decline in rod-mediated performance. ${ }^{12,13}$

It is believed that the macular pigment plays a crucial role in the retina as a powerful antioxidant and short-wavelength light filter, which may help protect the retinal pigment epithelium and photoreceptors from oxidative damage over a lifetime. ${ }^{14}$ This is of particular interest with regard to AMD, as it is hypothesised that one of the primary contributions to this condition is increasing retinal pigment epithelium dysfunction with age due to cumulative oxidative damage. ${ }^{15}$ It has been shown that lutein and zeaxanthin, two of three constituents of the macular pigment, are present in significant amounts in the rod outer segments. ${ }^{16,17}$ This finding leads to the hypothesis that the macular pigment may be involved in helping maintain normal rod physiology and subsequent rod-mediated function in ageing. Indeed, Stringham et al (2015) recently reported that in a small group (27 participants) of younger healthy individuals both dark adaptation recovery time and absolute scotopic thresholds were associated with macular pigment levels over a central retinal area. ${ }^{18}$ Patryas et al. (2014) also found a weak positive association (which was not statistically significant) between the slope of S2 and S3 portions of the dark adaptation curve at 11 degrees eccentricity and central macular pigment estimates using 33 participants. ${ }^{19}$ Conversely however, in a recent study Zarubina et al., 2018 showed in a large sample of healthy older individuals (participants=225; mean age 68.2 years) that RMDA was not associated with central macular pigment levels. ${ }^{20}$ They conclude that such a finding fits with the aforementioned vascular-metabolic theory for RMDA increases and query the basis for expecting a relationship between RMDA and macular pigment given that the macular pigment level in rod outer segments is low relative to the high levels in the foveal centre. However, they do acknowledge that further research on the distribution of the macular pigment within different retinal cells is required. 
None of these previous studies included participants with any clinical signs of AMD. No published information on the relationship between RMDA and macular pigment levels exists in individuals with AMD. If RMDA in AMD is associated with macular pigment levels it would be of particular interest to clinicians as the macular pigment can potentially be enhanced through dietary and lifestyle changes and by supplementation. Indeed, AREDS2 suggested that it may be appropriate to replace beta carotene with lutein and zeaxanthin in AREDS type antioxidant supplements for those individuals with intermediate AMD. ${ }^{21}$

The objective of this study was to establish whether or not RMDA is associated with macular pigment levels in intermediate stage AMD patients, who have an increased risk of developing exudative AMD over time. ${ }^{22}$

\section{METHODS}

This cross-sectional observational study was undertaken at the Vision Science research laboratory at the University of Ulster, which is a regional University in the United Kingdom. Participants were recruited by advertisement from the public optometry clinic in Ulster University and through local advertisement with macular disease support groups and optometry practices. Ethical approval for the study was obtained from the University of Ulster Research Ethics Committee and informed written consent was obtained from all participants after explanation of the nature and possible consequences of the study in accordance with the tenets of the Declaration of Helsinki.

\section{Participants}

Intermediate AMD

All individuals had a visual acuity of at least $0.40 \log$ MAR (>6/15 Snellen) with best spectacle lens correction of between $\pm 6 \mathrm{DS}$ and less than $3 \mathrm{DC}$ in the study eye. After the visual function testing (described below) was completed, dilated $30^{\circ}$ colour fundus photographs centred on the macula were taken (Canon CR-DGi, Uxbridge, UK). Each photograph was examined independently by two experienced optometrists to confirm participants had intermediate AMD as defined using an internationally accepted grading scale. ${ }^{23}$ Intermediate AMD was defined as any medium sized drusen (63 to $125 \mu \mathrm{m}$ ) with pigmentary abnormalities associated with AMD or any large drusen $(>125 \mu \mathrm{m})$ within two disc diameters of the fovea. In addition, all participants underwent ocular coherence tomography scanning using the Fast Retinal protocol of the retinal application of the Heidelberg Spectralis with TruTrack eye tracking technology (Heidelberg, Germany). This is a macular volume scan centred on the fovea with 19 equally separated horizontal line scans covering a 20 by 20 degrees square area, with 8 averaged frames for each 
line to reduce noise and enhance resolution. Images were assessed to exclude anyone with any other co-existing abnormality, such as epi-retinal membrane or significant vitreomacular traction, which could contribute to vision loss other than the intermediate AMD. Participants were excluded if they had any other co-existing eye disease (such as glaucoma or diabetic retinopathy) which would impact on the findings.

\section{Healthy individuals}

All participants had a best corrected visual acuity of 0.20 logMAR (Snellen 6/9.5) or better in the study eye, refractive error between +6DS and -6DS with <3.00DC and normal retinal health based on fundus photography and OCT examination. Anyone with amblyopia in the study eye, advanced AMD in their fellow eye or a history of systemic conditions which may affect vision were excluded. None of the older participants had any visually significant age-related cataract as judged on dilated slit-lamp examination.

\section{Visual function}

Monocular logMAR visual acuity was determined, after a full subjective refraction, with a BaileyLovie chart with distance prescription, with credit given for each correct letter read. Monocular contrast sensitivity was determined using the Mars Letter Contrast Sensitivity chart (Mars Perceptrix, Chappaqua, NY, US). Participants were instructed to read the letter chart, which was positioned at $50 \mathrm{~cm}$ with the appropriate near correction in place, until the letters became indistinguishable. The log contrast sensitivity value is determined as the final letter correctly identified before the patient misidentifies two consecutive letters, with incorrect responses prior to this taken off the final log contrast sensitivity value. For individuals with bilateral intermediate AMD the study eye was that with the better logMAR acuity. If acuity was equal in both eyes, then the eye with the better contrast sensitivity value was used for testing.

\section{Macular Pigment Optical Density}

Macular pigment optical density was estimated in the study eye at 0.5 degrees from the foveal centre using a heterochromatic flicker photometry based densitometer instrument (Macular Metrics, Rehoboth, MA, USA) which has been described in detail in several previous studies measuring macular pigment in normal, ${ }^{24,25}$ and AMD patients (using an adapted version). ${ }^{26}$

Firstly, several practice thresholds were measured to ensure the participant understood the task to be performed. Under monocular viewing conditions in a dim room the participant was asked to fixate on the centre of a flickering target, a solid $1^{\circ}$ diameter target comprised of two alternating wavelengths $(460 \mathrm{~nm}$ and $560 \mathrm{~nm})$ displayed on a $6^{\circ}$ blue background $(470 \mathrm{~nm})$. The participant was then instructed to adjust the radiance of the two alternating lights, which were offset from 
threshold to ensure there was clear visible flicker to begin with, until the point where the test target appeared stationary, or had minimal flicker. The two wavelengths of light were inversely yoked to maintain a constant brightness and threshold was measured using the optimal flicker frequency for that individual (measured before thresholds were taken). The task was repeated using a disc of $2^{\circ}$ in diameter presented at $7^{\circ}$ eccentricity from a central red fixation point subtending 5 minutes of arc. Four thresholds at each retinal location were taken for calculating the macular pigment optical density which was calculated using the following formula: Macular pigment optical density $=\log \left(R_{460} / R 460_{P}\right)-\log \left(R 560_{F} / R 560_{P}\right)$ where $R 460$ is the radiance of the $460 \mathrm{~nm}$ light and $\mathrm{R} 560$ the radiance of the $560 \mathrm{~nm}$ light at the foveal (F) and peripheral $(P)$ locations when no/minimal flicker is perceived.

\section{Rod-mediated function}

RMDA was assessed with the AdaptDx (MacuLogix, Hummelstown, PA) which measures the rod intercept time. This is the time from the onset of a reduced retinal bleach to reach a criterion light level on the dark adaptation curve, where vision is completely rod mediated. Those with better rod recovery will have a shorter rod intercept time than those with impaired rod function, allowing rod-mediated performance to be more easily compared between individuals using one outcome parameter. The instrument has been used in several studies looking at rod mediated function in healthy individuals and in various stages of AMD. ${ }^{7-10,27,28}$

For this measure the pupil of each participant's test eye was dilated to at least $6 \mathrm{~mm}$ in diameter using $0.5 \%$ Tropicamide to eliminate the effects of pupil size on the rod intercept time measure. Participants were instructed to fixate on a red fixation light located 5 degrees superiorly from the centre of the screen for the duration of the test and advised that a single spot stimulus would appear directly below this point throughout the test. Patient fixation was assessed throughout using an infrared camera which displayed the patients eye on the operating screen. Corrective lenses suitable for the $30 \mathrm{~cm}$ viewing distance were positioned in front of the test eye using the built-in lens holder provided by the instrument to correct for optical blur with the other eye being covered by an opaque patch.

A $505 \mathrm{~nm}$ photoflash (0.8ms duration, $1.8 \times 10^{4} \mathrm{scot} \mathrm{cd} / \mathrm{m}^{2}$ intensity) provided a $\sim 76 \%$ rod bleach equivalent over $\mathrm{a} 4^{\circ}$ area of retina centred at $5^{\circ}$ eccentricity directly below the fixation light. Threshold sensitivity measurements began immediately after at this location using a $2^{\circ}$ diameter circular target (wavelength $505 \mathrm{~nm}$ ) with a 3 down/1-up modified staircase estimate procedure. Participants were given 2 seconds to respond if the stimulus was visible using a response button. Fixation error rate was calculated as a percentage of the number of unreliable thresholds (threshold deviated from the previous measurement by $\geq 0.2$ log units) compared with 
the total number of thresholds. All individuals had a fixation loss of less than $35 \%$ (average fixation loss was $14.7 \%$ ). Testing concluded when the subject's sensitivity recovered to $5 \times 10^{-3}$ scotopic $\mathrm{cd} / \mathrm{m}^{2}$ (3.0 log units attenuation of the stimulus) determined by the internal software of the instrument, as this threshold level is considered to be completely mediated by rod function. The test could continue to up to 45 minutes at which point it was terminated if the recovery threshold had not been reached (a 45 minute rod intercept time was assigned to those participants for statistical analysis).

\section{Statistical analysis}

Statistical analyses were performed with SPSS software version 22 (IBM Corporation, Armonk, NY). Macular pigment optical density was measured in log units and the rod intercept time in minutes. Shapiro-Wilks tests showed that the macular pigment estimates were normally distributed for both groups $(p>0.05)$ but that the rod intercept time in the intermediate AMD group was not normally distributed $(p<0.05)$. Differences in age, logMAR acuity, contrast sensitivity and macular pigment optical density between the two groups were analysed using independent $t$ tests. Correlation between any of these variables was examined using the Pearson correlation coefficient $\left(r_{p}\right)$. The difference in the rod intercept time between the groups was analysed using the Mann-Whitney $U$ test. Correlation between the rod intercept time and any other variable in the intermediate stage AMD group was examined using the Spearman's rank correlation coefficient $\left(r_{s}\right)$. The study had better than $80 \%$ power to detect a large correlation between macular pigment optical density and the rod intercept time ( $r=0.52$ or better) at the 0.05 significance level in the intermediate AMD group, which was the primary objective of the study.

\section{RESULTS}

Twenty-seven individuals with intermediate AMD (12 males, 15 females; mean age 76.7 years (range 63-88 years)) were recruited to the study. Eight of these individuals had early AMD in their fellow eye, while nine had intermediate stage and ten had late stage AMD. Twenty-three older individuals free from any ocular disease (10 males, 13 females; mean age 74.0 years (range 68-86 years) were recruited for comparison with the AMD group. Two of these individuals had early AMD in their fellow eye while all others had no sign of AMD in their fellow eye. Table one summarises the visual function measures for those with intermediate stage AMD and the normal group. This shows that for the two age-matched groups all measures of visual function were significantly poorer in the intermediate AMD group $(p<0.05)$ but that there was no statistically significant difference in macular pigment optical density between the two groups ( $p>0.05$ ). When those individuals in the AMD group who were taking any form of supplement containing one or more of the retinal carotenoids (number=13) were removed from the analysis there remained no statistically significant difference in macular pigment optical density levels 
between the healthy normal group and the intermediate AMD group ( 0.44 versus $0.41 \log$ units; $p=0.62$ ). Smoking status (never a smoker versus previous smoker) did not have any statistically significant effect on macular pigment values in either the AMD group $(p=0.23)$ or the normal group ( $p=0.36$ ). The rod intercept time for the intermediate AMD individuals was, on average, three times longer than that of the normal individuals indicating that a significant deficit in rod-mediated performance exists (32.9mins versus 10.7 mins). While there was significant overlap in logMAR acuity values between the two groups only two intermediate AMD individuals had a rod-intercept value which fell within the range of the values found in the normative group.

There was no statistically significant correlation between the rod intercept time and the level of macular pigment in either the normal group $\left(r_{p}=-0.16, p=0.46\right)$ or the AMD group $\left(r_{s}=-0.04, p=0.85\right)$ (Figure One). When the individuals with previous late AMD in the fellow eye were removed from the intermediate AMD analysis no statistically significant relationship was found between macular pigment optical density and the rod intercept time $\left(r_{s}=-.13, p=0.63\right)$. There was no statistically significant relationship between the rod intercept time and logMAR acuity in the intermediate AMD group $\left(r_{s}=0.33, p=0.09\right)$ however there was a statistically significant relationship between the rod intercept time and contrast sensitivity $\left(r_{s}=-0.41, p=0.03\right)$. The rod intercept time was higher in those individuals who had late-stage AMD in the fellow eye, compared those without late AMD in the fellow eye, but this failed to reach statistical significance (38.6 versus 29.6 mins; $p=0.05$ ). There was no significantly significant difference in acuity, CS or age between these two groups ( $p>0.05$ for all). Macular pigment optical density was significantly higher in those individuals who had late stage AMD in the fellow eye ( 0.55 versus 0.40 log units, $\mathrm{p}=0.04)$. However, eighty percent of those participants who had late-stage AMD in their fellow eye took some form of supplementation containing lutein and/or zeaxanthin (in comparison to $29 \%$ of those without late AMD in the fellow eye).

Eleven individuals reached the maximum rod intercept time of 45 minutes without reaching the rod recovery criterion point (seven of these individuals had late AMD in the fellow eye). There was no statistically significant difference in macular pigment levels between those who reached the maximum time (mean rod intercept time $=45$ mins, mean macular pigment $=0.45$ ) in comparison to all of the other individuals in the AMD group (mean $=24.6 \mathrm{mins}$, mean macular pigment $=0.45(t=0.09, p=0.93)$.

\section{DISCUSSION}

Several recent studies have investigated the relationship between RMDA and macular pigment levels in healthy individuals free from ocular disease. ${ }^{18-20}$ The largest of these three studies showed no statistically significant relationship between RMDA, measured using the AdaptDx, and 
macular pigment optical density estimated using a heterochromatic flicker photometry based instrument. ${ }^{20}$ This current study, using the same retinal locations for RMDA and macular pigment measures as Zarubina et al. 2018, has shown that there is no statistically significant association between RMDA and macular pigment in individuals with intermediate stage AMD. In this study we minimised any influence age-related optical changes in the eye may have on RMDA by dilating all participants, correcting for refractive error and by including only those free from cataract. Therefore, the measurement of RMDA using the AdaptDx rod intercept time primarily reflects the function of the underlying rod photoreceptors. The rod intercept time measures found in this study are similar to those reported in a previous study with AMD participants using similar methods. ${ }^{8}$

It could be argued that if an individual had a significant amount of macular pigment at 5 degrees eccentricity it could have a detrimental effect on the rod intercept time simply by reducing the visibility of the $505 \mathrm{~nm}$ light due to the spectral absorbance profile of the macular pigment ${ }^{29}$. However, significant amounts of macular pigment have been measured in only a very small percentage of individuals at 5 degrees eccentricity, ${ }^{30,31}$ with the vast majority displaying no measurable macular pigment at this retinal location. The very maximum amount of macular pigment measured in any individual reported in the literature is less than 0.1 log units at 5 degrees from the foveal centre ${ }^{31}$. Wooten and Hammond, 1992 show that for this level of macular pigment there would still be $90 \%$ transmittance of the $505 \mathrm{~nm}$ stimuli for measuring the rod intercept time (see Figure 7 in this publication) ${ }^{32}$. Therefore, we can be fairly certain that the 5 degrees location chosen did not inadvertently remove any relationship between these functional measures. Although two previous studies in normal individuals used a more eccentric retinal location for measuring rod-mediated performance (both beyond 10 degrees eccentricity), ${ }^{18,19}$ we believe that a more eccentric location for the rod intercept time measure would have made the task considerably more difficult for the participants, resulting in less reliable measures.

One assumption of this study is that the single central measure of macular pigment reported in this study is an appropriate reflection of the overall level of macular pigment within the retina. Although macular pigment has been found in significant amounts in rod outer segments, ${ }^{16,17}$ most of the macular pigment has been shown to be concentrated in the pre-receptoral Muller cells in the foveal centre. This location lends itself more to the idea that the macular pigment may have a stronger relationship with cone mediated visual functions, and there is some evidence to suggest that this might indeed be the case. ${ }^{33}$

As the study included 27 individuals with intermediate AMD we only had sufficient statistical power to detect a large correlation ( $>0.52$ ) between these two measures. However, Figure 1 suggests that a statistically significant relationship between these two measures would be unlikely to emerge even with more participants. Even those individuals who could not reach the 
arbitrary rod intercept time threshold scotopic sensitivity within 45 minutes did not have a level of macular pigment that was significantly different to those who reached this point in a much faster time. Those that reached the arbitrary cut-off rod intercept time also had macular pigment values across the whole range measured (0.14-0.85 log units).

The protective effect of the macular pigment on the risk of developing AMD remains inconclusive with evidence for and against this theory. ${ }^{14,34,35}$ In this study, with a relatively small number of participants, there was no significant difference in the amount of macular pigment measured in those with intermediate AMD compared to the age-matched healthy individuals. Of course, it must be recognised that the control group in this study was simply based on the observation that these individuals were free from fundal signs of AMD. We didn't collect any information on dietary intake or assess serum levels of carotenoids. Therefore, it must be considered that the dietary intake (and supplement intake) could be significantly different between the two groups hiding any true baseline differences in macular pigment levels earlier in life. In addition to this the disease process itself may lead to loss of the macular pigment which is not accounted for using such methods. Several previous studies have investigated macular pigment levels in various stages of AMD and while some have found a significant reduction in macular pigment in AMD patients, ${ }^{36}$ several others have not. ${ }^{37,38}$ Very limited data exists on baseline levels of macular pigment levels prior to the clinical appearance of macular degeneration. ${ }^{39}$ Until substantial data on predisease macular pigment levels is available for individuals who subsequently develop AMD, the true impact of the macular pigment on disease risk will continue to be debated.

In conclusion the results of this study do not support the hypothesis that higher macular pigment is associated with improved rod-mediated performance or that higher levels protect rod-mediated function in intermediate AMD.

\section{ACKNOWLEDGEMENTS}

Supported by a Research Grant from the Macular Society (UK). 


\section{REFERENCES}

1. Lamb TD, Pugh EN,Jr. Dark adaptation and the retinoid cycle of vision. Prog Retin Eye Res 2004;23:307-380.

2. Jackson GR, Owsley C, McGwin G,Jr. Aging and dark adaptation. Vision Res 1999;39:3975-82.

3. Owsley C, Jackson GR, Cideciyan AV et al. Psychophysical evidence for rod vulnerability in age-related macular degeneration. Invest Ophthalmol Vis Sci 2000;41:267-73.

4. Owsley C, McGwin G,Jr, Jackson GR et al. Cone- and rod-mediated dark adaptation impairment in age-related maculopathy. Ophthalmology 2007;114:1728-1735.

5. Owsley C, Jackson GR, White $M$ et al. Delays in rod-mediated dark adaptation in early age-related maculopathy. Ophthalmology 2001;108:1196-202.

6. Dimitrov PN, Guymer RH, Zele AJ et al. Measuring rod and cone dynamics in agerelated maculopathy. Invest Ophthalmol Vis Sci 2008;49:55-65.

7. Owsley C, McGwin G,Jr, Clark ME et al. Delayed rod-mediated dark adaptation is a functional biomarker for incident early Age-Related Macular Degeneration. Ophthalmology 2016;123:344-351.

8. Flamendorf J, Agron E, Wong WT et al. Impairments in dark adaptation are associated with age-related macular degeneration severity and reticular pseudodrusen. Ophthalmology 2015;122:2053-2062.

9. Jackson GR, Scott IU, Kim IK et al. Diagnostic sensitivity and specificity of dark adaptometry for detection of age-related macular degeneration. Invest Ophthalmol Vis Sci 2014;55:1427-31.

10. Jackson GR, Clark ME, Scott IU et al. Twelve-month natural history of dark adaptation in patients with AMD. Optom Vis Sci 2014;91:925-31.

11. Curcio CA, Owsley C, Jackson GR. Spare the rods, save the cones in aging and agerelated maculopathy. Invest Ophthalmol Vis Sci 2000;41:2015-8.

12. Pokorny J, Smith VC, Lutze M. Aging of the human lens. App/ Opt 1987;26:14371440.

13. IJspeert JK, de Waard PW, van den Berg TJ et al. The intraocular straylight function in 129 healthy volunteers; dependence on angle, age and pigmentation. Vision Res 1990;30:699-707.

14. Loane $\mathrm{E}$, Kelliher $\mathrm{C}$, Beatty $S$ et al. The rationale and evidence base for a protective role of macular pigment in age-related maculopathy. Br J Ophthalmol 2008;92:11638. 
15. Suzuki M, Kamei $\mathrm{M}$, Itabe $\mathrm{H}$ et al. Oxidized phospholipids in the macula increase with age and in eyes with age-related macular degeneration. Mol Vis 2007;13:772-778.

16. Rapp LM, Maple SS, Choi JH. Lutein and zeaxanthin concentrations in rod outer segment membranes from perifoveal and peripheral human retina. Invest Ophthalmol Vis Sci 2000;41:1200-9.

17. Sommerburg OG, Siems WG, Hurst JS et al. Lutein and zeaxanthin are associated with photoreceptors in the human retina. Curr Eye Res 1999;19:491-5.

18. Stringham JM, Garcia PV, Smith PA et al. Macular Pigment and Visual Performance in Low-Light Conditions. Invest Ophthalmol Vis Sci 2015;56:2459-2468.

19. Patryas L, Parry NR, Carden D et al. The association between dark adaptation and macular pigment optical density in healthy subjects. Graefes Arch Clin Exp Ophthalmol 2014;252:657-63.

20. Zarubina AV, Huisingh CE, Clark ME et al. Rod-mediated dark adaptation and macular pigment optical density in older adults with normal maculas. Curr Eye Res 2018;43:913-920.

21. Chew EY, Clemons TE, Sangiovanni JP, et al. Lutein and zeaxanthin and omega-3 fatty acids for age-related macular degeneration: the Age-Related Eye Disease Study 2 (AREDS2) randomized clinical trial. JAMA 2013;309:2005-15.

22. Joachim N, Mitchell P, Burlutsky $G$ et al. The incidence and progression of agerelated macular degeneration over 15 Years: The Blue Mountains Eye Study. Ophthalmology 2015;122:2482-2489.

23. Ferris FL,3rd, Wilkinson $\mathrm{CP}$, Bird $\mathrm{A}$ et al. Clinical classification of age-related macular degeneration. Ophthalmology 2013;120:844-51.

24. Wooten BR, Hammond BR, Land RI et al. A practical method for measuring macular pigment optical density. Invest Ophthalmol Vis Sci 1999;40:2481-9.

25. Beirne RO. The macular pigment optical density spatial profile and increasing age. Graefes Arch Clin Exp Ophthalmol 2014;252:383-388.

26. Stringham JM, Hammond BR, Nolan JM et al. The utility of using customized heterochromatic flicker photometry (cHFP) to measure macular pigment in patients with age-related macular degeneration. Exp Eye Res 2008;87:445-53.

27. Jackson GR, Edwards JG. A short-duration dark adaptation protocol for assessment of age-related maculopathy. J Ocul Biol Dis Infor 2008;1:7-11.

28. Owsley C, Huisingh C, Jackson GR et al. Associations between abnormal rodmediated dark adaptation and health and functioning in older adults with normal macular health. Invest Ophthalmol Vis Sci 2014;55:4776-4789. 
29. Snodderly DM, Brown PK, Delori FC et al. The macular pigment. I. Absorbance spectra, localization, and discrimination from other yellow pigments in primate retinas. Invest Ophthalmol Vis Sci 1984;25:660-73.

30. Hammond BR, Wooten BR, Snodderly DM. Individual variations in the spatial profile of human macular pigment. J Opt Soc Am A Opt Image Sci Vis 1997;14:1187-96.

31. Robson AG, Moreland JD, Pauleikhoff D et al. Macular pigment density and distribution: comparison of fundus autofluorescence with minimum motion photometry. Vision Res 2003;43:1765-1775.

32. Wooten BR, Hammond BR. Macular pigment: influences on visual acuity and visibility. Prog Retin Eye Res 2002;21:225-40.

33. Loughman J, Akkali MC, Beatty $S$ et al. The relationship between macular pigment and visual performance. Vision Res 2011;50:1249-56.

34. Weale RA. Guest editorial: Notes on the macular pigment. Ophthalmic Physiol Opt 2007;27:1-10.

35. Barker FM,2nd, Snodderly DM, Johnson EJ et al. Nutritional manipulation of primate retinas, $\mathrm{V}$ : effects of lutein, zeaxanthin, and $\mathrm{n}-3$ fatty acids on retinal sensitivity to blue-light-induced damage. Invest Ophthalmol Vis Sci 2011;52:3934-3942.

36. Kaya S, Weigert G, Pemp B et al. Comparison of macular pigment in patients with age-related macular degeneration and healthy control subjects - a study using spectral fundus reflectance. Acta Ophthalmol 2012;90:e399-403.

37. Dietzel M, Zeimer M, Heimes B et al. Determinants of macular pigment optical density and its relation to age-related maculopathy: results from the Muenster Aging and Retina Study (MARS). Invest Ophthalmol Vis Sci 2011;52:3452-3457.

38. LaRowe TL, Mares JA, Snodderly DM et al. Macular pigment density and age-related maculopathy in the Carotenoids in Age-Related Eye Disease Study. An ancillary study of the women's health initiative. Ophthalmology 2008;115:876-883.e1.

39. Kanis MJ, Berendschot TT, van Norren D. Influence of macular pigment and melanin on incident early AMD in a white population. Graefes Arch Clin Exp Ophthalmol 2007;245:767-773. 


\begin{tabular}{|c|c|c|c|}
\hline & \begin{tabular}{|c|} 
Normals \\
(number $=23$ ) \\
Pseudophakic $=5$ \\
Former smoker $=10$ \\
On supplementation with \\
lutein or zeaxanthin $=3$
\end{tabular} & $\begin{array}{c}\text { Intermediate AMD } \\
\text { (number }=27) \\
\text { Pseudophakic }=2 \\
\text { Former smoker }=7 \\
\text { On supplementation with } \\
\text { lutein or zeaxanthin }=13\end{array}$ & Significance \\
\hline Age (years) & $\begin{array}{c}74.0 \text { years } \\
\text { (range 68-86) }\end{array}$ & $\begin{array}{c}76.7 \text { years } \\
\text { (range 63-88) }\end{array}$ & $p=0.10$ \\
\hline LogMAR acuity & $\begin{array}{c}0.04( \pm 0.10) \\
\text { Range: }-0.18-+0.20\end{array}$ & $\begin{array}{c}0.17( \pm 0.13) \\
\text { Range: }-0.06-+0.40\end{array}$ & $p=<0.01$ \\
\hline Contrast sensitivity & $\begin{array}{c}1.46( \pm 0.14) \\
\text { Range: } 1.20-1.72\end{array}$ & $\begin{array}{c}1.32( \pm 0.18) \\
\text { Range: } 0.96-1.64\end{array}$ & $p=<0.01$ \\
\hline $\begin{array}{l}\text { Macular pigment } \\
\text { optical density }\end{array}$ & $\begin{array}{c}0.44( \pm 0.18) \\
\text { Range: } 0.09-0.76\end{array}$ & $\begin{array}{c}0.45( \pm 0.20) \\
\text { Range: } 0.14-0.85\end{array}$ & $p=0.85$ \\
\hline $\begin{array}{l}\text { Rod intercept time } \\
\text { (mins) }\end{array}$ & $\begin{array}{c}10.7( \pm 3.2) \\
\text { Range: } 5.64-16.03\end{array}$ & $\begin{array}{c}32.9( \pm 11.5) \\
\text { Range: } 12.06-45\end{array}$ & $p=<0.01$ \\
\hline
\end{tabular}

Table 1. Participant characteristics and visual function measures for the normal group and those with intermediate AMD. Differences between the two groups was analysed using an independent t-test for all variables except the rod intercept time where a MannWhitney U test was used. 
Figure

Figure 1: Rod intercept time values at 5 degrees eccentricity (superior retina/inferior field) versus macular pigment optical density levels at 0.5 degrees eccentricity from the foveal centre for healthy normal participants (open circles) and those with intermediate stage AMD (closed circles). Two sets of individuals in the intermediate stage AMD group, who all had a rod intercept time of 45 minutes, had exactly the same macular pigment optical density values ( 0.14 and $0.25 \mathrm{log}$ units).There was no statistically significant relationship between the two measures in either group $(p>0.05)$.

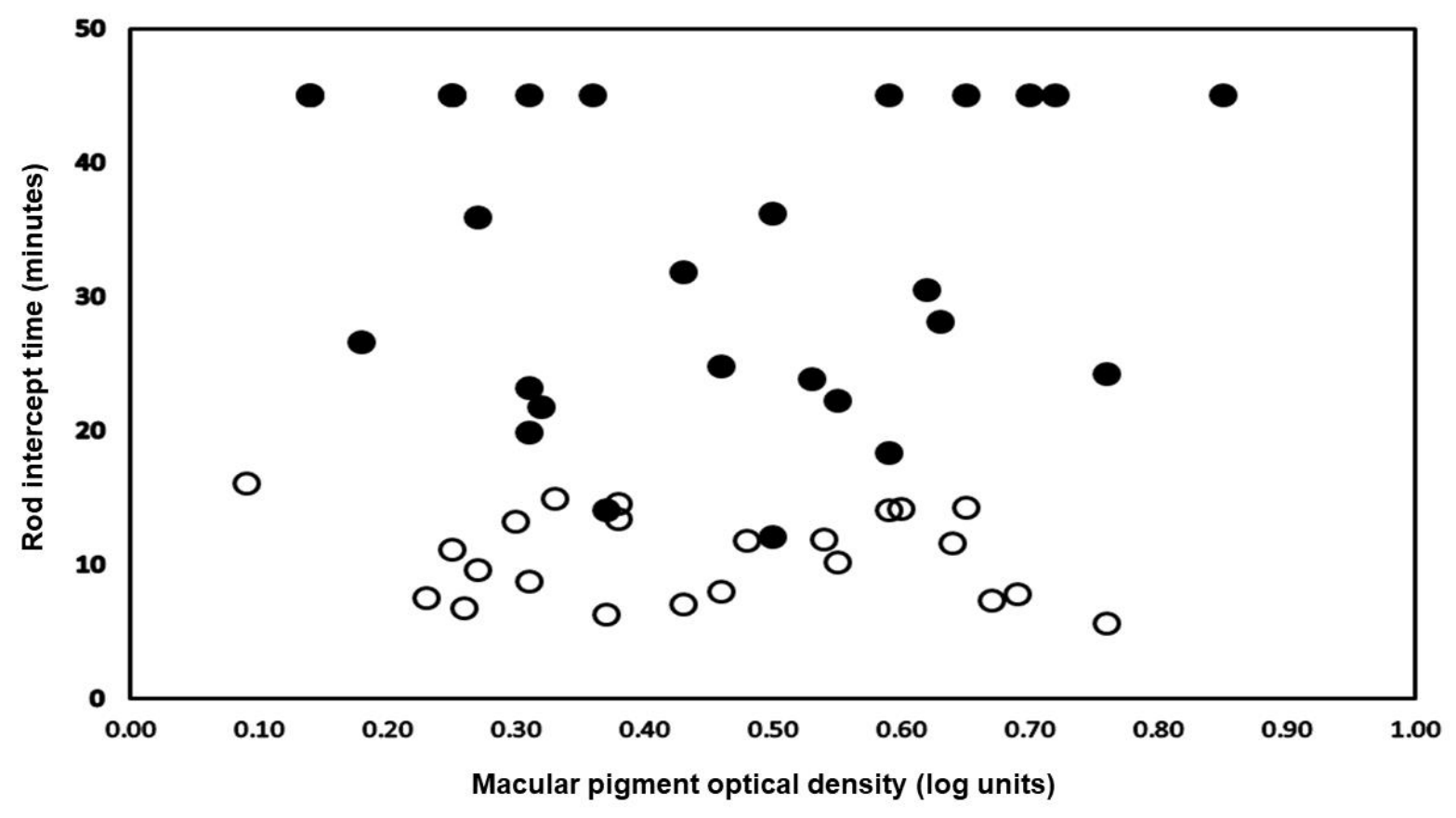

